\title{
Erratum to: Nutritional \\ Considerations After \\ Gastrectomy and Esophagectomy \\ for Malignancy
}

Amelia Baker, RD, LD, CNSC ${ }^{*} 1$

Leigh-Anne Wooten, MS, RD, LD

Michele Malloy, MALIS

\section{Address}

${ }^{* 1}$ Georgetown University Hospital, Washington, DC 20007-2113, USA

Email:axb43@gunet.georgetown.edu

${ }^{2}$ Georgetown University Medical Center, Washington, DC 20007-2113, USA

Published online: 1 March 2011

(C) Springer Science+Business Media, LLC 2011

Erratum to: Curr. Treat. Options in Oncol.

DOI 10.1007/s11864-010-0134-0

This article did not contain a conflict of interest statement upon online publication. All authors had completed a disclosure form stating that they did not have any potential conflicts of interest relevant to this article. An error occurred on authors' affiliation Georgetown University Hospital zip code should be 20007-2113. Michele Malloy is employed by Georgetown University Medical Center, not Georgetown University. 